\title{
Membrane tension feedback on shape and motility of eukaryotic cells
}

\author{
Benjamin Winkler ${ }^{\mathrm{a}}$, Igor S. Aranson ${ }^{\mathrm{b}, \mathrm{c}, 1}$, Falko Ziebert ${ }^{\mathrm{a}, 2}$ \\ ${ }^{a}$ Physikalisches Institut, Albert-Ludwigs-Universität, 79104 Freiburg, Germany \\ ${ }^{b}$ Materials Science Division, Argonne National Laboratory, 9700 S. Cass Avenue, \\ Argonne, IL 60439, USA \\ ${ }^{c}$ Engineering Sciences and Applied Mathematics, Northwestern University, 2145 \\ Sheridan Road, Evanston, IL 60202, USA
}

\begin{abstract}
In the framework of a phase field model of a single cell crawling on a substrate, we investigate how the properties of the cell membrane affect the shape and motility of the cell. Since the membrane influences the cell dynamics on multiple levels and provides a nontrivial feedback, we consider the following fundamental interactions: (i) the reduction of the actin polymerization rate by membrane tension; (ii) area conservation of the cell's two-dimensional cross-section vs. conservation of the circumference (i.e. membrane inextensibility); and (iii) the contribution from the membrane's bending energy to the shape and integrity of the cell. As in experiments, we investigate two pertinent observables - the cell's velocity and its aspect ratio. We find that the most important effect is the feedback of membrane tension on the actin polymerization. Bending rigidity has only minor effects, visible mostly in dynamic reshaping events, as exemplified by collisions of the cell with an obstacle.
\end{abstract}

Keywords: motility, bending rigidity, cytoskeleton

Email addresses: aronson@anl.gov (Igor S. Aranson), falko.ziebert@physik.uni-freiburg.de (Falko Ziebert)

${ }^{1}$ phone: +1 630252 9725; fax: +1 6302527777

2phone: +49761203 97779; fax: +497612035855

Preprint submitted to Physica D

September 10, 2015

(C) 2015. This manuscript version is made available under the Elsevier user license http://www.elsevier.com/open-access/userlicense/1.0/ 


\section{Introduction}

Motility of cells crawling on substrates attracts substantial interest among biologists, physicists, mathematicians and material scientists alike $[1,2,3,4$, 5]. Cell motility is a fundamental phenomenon that is crucial for a variety of biological processes, from morphogenesis to immune response [6, 7]. It is also involved in pathologies like cancer growth and metastasis [6]. Like swimming microorganisms, crawling motile cells are natural and interesting realizations of active, self-propelled systems, displaying self-organized dynamics [8], flows, as well as intriguing collective effects [9]. Moreover, motile cells and living tissues are inspiring novel adaptive materials with intricate properties like active visco-elastic response [10] and self-healing [11]. Cellular materials, responding on the topography, elasticity, and surface chemistry of the substrate they are in contact with, currently inspire microstructured design strategies for cell sorting and guiding [12].

The main processes involved in the motion of eukaryotic cells (such as keratocytes, fibroblasts or neutrophils) are the following: the generation of a propulsive force by actin polymerization against the cell's membrane, the formation of adhesive contact to the substrate to transfer this propulsion force and to move forward, and finally, the action of molecular motors in determining the cell's polarity and to retract the rear of the cell [13]. All these processes have been modeled in some detail, and models for whole moving cells have been recently developed $[14,15,16,17,18]$. However, there is another important player in the game, that has been neglected (or its consequences not yet thoroughly studied) in most of the modeling approaches: namely, the membrane enclosing the cell. The cell membrane represent a movable interface which constitutes an intricate theoretical and numerical problem. In addition, membrane tension leads to a global force feedback, affecting the propulsion by ratcheting the actin filaments. Moreover, membrane bending rigidity may be relevant in some cases, especially for cell collisions with other cells or obstacles.

The first detailed experimental study on the effects of membrane tension on spreading cells (fibroblasts) dates back no longer than in 2000 [19]. There, an inverse relation between spreading/lamellipodium extension and membrane tension was found: lowering the membrane tension by adding detergents (deoxycholic acid) or lipids led to an increased spreading and extension, while an increase in tension by placing cells in a hypotonic medium reduced both effects. The authors concluded that membrane tension may 
constitute a global coupling involved in determining both the cell's shape and the propulsion dynamics, cf. also the recent reviews [20, 21]. The effect of membrane tension was studied also for neutrophils, both during pseudopod formation and for fully developed motion [22], for spreading fibroblasts [23], as well as for moving keratocytes [24]. Some of the observed effects include: (i) increased membrane tension can cause leukocytes to stop moving [22]; (ii) reducing tension can stimulate moving keratocytes to develop several fronts [24]; (iii) softening the cell membrane does not affect the velocity of keratocytes [24, 25], it only increases the retrograde flow of actin towards the cell's interior.

Membrane tension have been recently taken into account, for instance, in the one-dimensional model for growth cones [26], as well as the steady state. Force balance-based model in [27] includes also an explicit adhesion dynamics between the actin cortex and the membrane. Very recently, tension gradients and flows inside the membrane were addressed [28, 29]. However, these models do not take shape changes into account, obviously an important aspect of the membrane's feedback. As a result, they can not properly describe the onset/cessation of motion. These two important aspects can be easily and inherently modeled within the phase field approach recently developed for motile cells $[16,17,30,31,32,33,34,35]$, self-propelled active droplets [36, 37, 38], synthetic polymeric capsules [11]. Here we include and study the most pertinent membrane effects - tension and its feedback on polymerization, as well as bending. The study is performed within a simple phase field approach for a moving cell.

\section{Phase field model for a crawling cell}

The phase field approach to cell motility has been recently reviewed in [39]. Instead of modeling the cell's interface (i.e. the membrane) explicitly, an auxiliary field, the phase field $\rho(x, y ; t)$, is introduced. It evaluates to $\rho=1$ within the cell and to $\rho=0$ outside the cell, with a smooth transition region in between describing the 'smeared' interface. The simplest implementation of the phase field approach is via scalar order parameter equation

$$
\partial_{t} \rho=-\frac{\delta F_{P}}{\delta \rho}, \text { where } F_{p}=\int\left[f(\rho)+D_{\rho}(\nabla \rho)^{2}\right] d x d y .
$$

Here $f(\rho)=\frac{\rho^{2}(1-\rho)^{2}}{4}$ is a double well potential with minima at $\rho=0$ and $\rho=1$ (the two 'phases'). The phase field free energy $F_{p}$ in addition includes 
a surface energy term penalizing interfaces. Equation (1) yields

$$
\partial_{t} \rho=D_{\rho} \Delta \rho-\rho(1-\rho)(\delta-\rho)=: \lambda,
$$

where $\delta=\frac{1}{2}$ is the 'pressure difference' between the two 'phases'. For $\delta=\frac{1}{2}$ the free energy of both phases is equal, and hence a planar interface connecting states $\rho=0$ and $\rho=1$ is stationary. In case $\delta$ deviates from this value, the interface moves either forward or backward, i.e. the cell expands or retracts.

We used this simple framework to model a moving cell [17] by coupling the phase field Eq. (2) to the polarization field p, describing the averaged local orientation of the actin filaments inside the cell:

$$
\begin{aligned}
\partial_{t} \rho & =D_{\rho} \Delta \rho-\rho(1-\rho)\left(\delta-\sigma|\mathbf{p}|^{2}-\rho\right)-\alpha \mathbf{p} \cdot \nabla \rho \\
\partial_{t} \mathbf{p} & =D_{p} \Delta \mathbf{p}-\beta \nabla \rho-\tau_{1}^{-1} \mathbf{p}-\tau_{2}^{-1}\left(1-\rho^{2}\right) \mathbf{p}-\gamma[(\nabla \rho) \cdot \mathbf{p}] \mathbf{p} .
\end{aligned}
$$

In this description, the $\alpha$-term models the propulsion of the cell's interface by the ratcheting of actin, and the $\sigma$-term accounts for acto-myosin contraction. In Eq. (4), the terms $D_{p} \Delta \mathbf{p}$ and $-\tau_{1}^{-1} \mathbf{p}$ describe diffusion of actin and its degradation (depolymerization) in the bulk of the cell, respectively. The term $-\beta \nabla \rho$ describes the creation of actin polarization at the cell membrane (directed normal to the interface) with polymerization rate $\beta$. The contribution $-\tau_{2}^{-1}\left(1-\rho^{2}\right) \mathbf{p}$ assures a vanishing polarization outside of the cell (where $\rho=0)$. Finally, $-\gamma[(\nabla \rho) \cdot \mathbf{p}] \mathbf{p}$ models the front-rear symmetry breaking induced by motors. For details we refer to [17, 39].

Since motile cells are rather thin (typical lamellipodium thicknesses are $200 \mathrm{~nm}$ ) the model is effectively two-dimensional, i.e. height averaged. In addition, keratocyte cells are known to preserve their contact area with the substrate. To describe this conservation of the cell's contact area, we introduced the following global constraint

$$
\delta=\delta_{V}=\frac{1}{2}+\mu_{V}\left[V(t)-V_{0}\right]
$$

Here $\mu_{V}$ is the stiffness of the constraint and the term in brackets is the difference between the current area (or $2 \mathrm{D}$ volume) $V(t)=\int \rho(t) d x d y$ and the prescribed area $V_{0}$. Note that, to avoid confusion, in the following area always corresponds to the 2D area of the cell's cross-section (corresponding in a $3 \mathrm{D}$ description to the cell's volume), while the membrane refers to 
the surface, i.e. circumference, of this cross-section (corresponding in a 3D description to the cell's surface area).

The position of the interface - which is identified with the cell membrane -

can be defined in the model by the contour at $\rho=\frac{1}{2}$. However, this interface is not an appropriate description for a cell membrane: it has neither membrane tension nor bending energy, but rather an (artificial) wall energy $\left(\propto \sqrt{D_{\rho}}\right)$ that is related to the Ginzburg-Landau-type free energy of the phase field, cf. Eq. (2).

Even more important in the context of cell motility is the fact that membrane tension counteracts the polymerization force of the actin filaments: polymerization rate and hence the cell's velocity decrease as a function of the counteracting force, as established theoretically on a single filament level by the Brownian ratchet model $[40,41]$. Although studies of single/few actin filaments polymerizing against a load are very difficult, this effect could also been established experimentally $[42,43,44]$. The membrane tension feedback on actin polymerization possibly not only leads to a change in the overall velocity of the cell, but also to a global feedback on the actin organization and a change in the overall shape of the cell.

\section{Membrane tension as a counteracting force to polymerization}

We will first focus on the effect of membrane tension on actin polymerization within the whole cell model described in the last section. To this purpose, we remove the - artificial - wall energy of the phase field, and add the restoring force of the membrane counteracting polymerization. For simplicity, we keep the simple volume conservation and ignore at first the effect of tension on the phase field, a limit corresponding to a strongly adhering cell that keeps its contact area constant. The effect of tension on the phase field is added and studied in the next section.

The wall energy of the phase field potential can be removed - to leading order in the interface width - via addition of the following term to the phase field equation $[45,46]: \partial_{t} \rho=\ldots+D_{\rho} c|\nabla \rho|$, where $c=c(x, y)$ is the curvature of the interface. The latter can be calculated from the local normal unit vector, which is determined by the phase field like $\mathbf{n}(x, y)=\frac{\nabla \rho}{|\nabla \rho|}$, via the geometric identity $c=-\nabla \cdot \mathbf{n}$. Note that with that definition, the normal vector points to the inside of the cell.

Second, we introduce the membrane tension $\zeta(x, y)$. In the Helfrich pic- 
ture, the membrane energy reads [46]

$$
E_{m b}=\int \zeta|\nabla \rho| d x d y+\frac{b}{2} \int c^{2}|\nabla \rho| d x d y
$$

The first term implements the surface area constraint (circumference in 2D), where $\zeta$ is the membrane tension, i.e. the Lagrangian multiplier associated with the constraint. The second contribution is the bending energy with the curvature $c$ already introduced above and $b$ the corresponding bending modulus. The restoring force resulting from this energy has been calculated in [47] and reads (with $\mathbf{n}$ and $\mathbf{t}$ the normal and tangential unit vectors with respect to the interface)

$$
\mathbf{F}_{m b}=\left[\zeta c \mathbf{n}-b\left\{\frac{c^{3}}{2}+\mathbf{t} \cdot \nabla(\mathbf{t} \cdot \nabla c)\right\} \mathbf{n}+(\mathbf{t} \cdot \nabla \zeta) \mathbf{t}\right]|\nabla \rho| .
$$

Herein, the first term is the effect of the tension, the second one the contribution from bending and the last one arises from possible variations in tension along the membrane (similar to the Marangoni effect in thermal convection [48]).

Since the polymerization is normal to the membrane, we use only the normal contribution $F=\mathbf{n} \cdot \mathbf{F}_{m b}$. In addition, one can estimate that the contribution to the restoring force from bending $(\propto b)$ is negligible, see 7 . Hence we simply obtain that at the membrane (where $|\nabla \rho|$ is non-vanishing) the restoring force in normal direction is given by $F=\zeta c$ : the force counteracting polymerization is proportional to the tension and the local curvature.

The simplest way to determine the value of the tension $\zeta$ is to assume that it is related to the overall relative excess circumference of the membrane (in 3D: surface area) via ${ }^{3}$

$$
\zeta=T \delta A=T \frac{A(t)-A_{0}}{A_{0}}, \text { where } A(t)=\int|\nabla \rho|(t) d x d y .
$$

Here $T$ is the membrane's compressibility modulus and $A_{0}$ is the cell's circumference in a reference state. For the latter we chose the stationary, round, non-moving state, as it has the smallest circumference.

\footnotetext{
${ }^{3}$ Since $\nabla \rho$ is nonzero only at the interface, $\int|\nabla \rho|(t) d x d y$ is (proportional to) the cell's circumference.
} 
Finally, we have to account for the feedback the membrane tension provides on the actin polymerization dynamics. In the simple model, the term $-\beta \nabla \rho$ in the equation for $\mathbf{p}$ described that at the position of the membrane (where $\nabla \rho$ is nonzero), actin is created with constant rate $\beta$ in the normal direction ${ }^{4}$. Here, we make use of the ratchet-like process of actin polymerizing against the membrane, where it had been shown that the polymerization rate $\beta$ decreases exponentially (in the simplest case) with the force

$$
\beta(F)=\beta \exp \left(-\frac{a F}{k_{B} T}\right)=\beta e^{-f_{0} \zeta c},
$$

where $k_{B} T$ is the thermal energy and $a$ is the size of the actin monomer $[40,41]$, which can be absorbed in the constant $f_{0}$. Note that the parameter $\beta$ associated with actin polymerization is not the free polymerization rate ${ }^{5}$, but the rate reduced by the offset tension present in the reference state, cf. Eq. (8). Correspondingly, the propulsion strength, $\alpha \mathbf{p}$, decreases too upon an increase in membrane tension, which is due to the smaller amount of overall actin polarization $\mathbf{p}$.

The generalized model then reads

$$
\begin{aligned}
& \partial_{t} \rho=D_{\rho} \Delta \rho-\rho(1-\rho)\left(\delta-\sigma|\mathbf{p}|^{2}-\rho\right)+D_{\rho} c|\nabla \rho|-\alpha \mathbf{p} \cdot \nabla \rho, \\
& \partial_{t} \mathbf{p}=D_{p} \Delta \mathbf{p}-\beta e^{-f_{0} \zeta c} \nabla \rho-\tau_{1}^{-1} \mathbf{p}-\tau_{2}^{-1}\left(1-\rho^{2}\right) \mathbf{p}-\gamma[(\nabla \rho) \cdot \mathbf{p}] \mathbf{p},
\end{aligned}
$$

with $\delta$ given by Eq. (5) and $c=-\nabla \cdot \mathbf{n}=-\nabla \cdot\left(\frac{\nabla \rho}{|\nabla \rho|}\right)$ the curvature as described above. For numerical reasons, the normal vector and the curvature can only be calculated in a 'tube' around the interface (using a threshold value for $\nabla \rho$ ). Upon multiplication with $|\nabla \rho|$ the respective terms nevertheless lead to smooth contributions at the interface, where they are needed.

Results for steady moving cells. We investigated the behavior of the following quantities that can be easily measured in experiments: the aspect ratio of the cell as a measure for the shape change, the cell's velocity and its

\footnotetext{
${ }^{4}$ Note that $\nabla \rho$ is negative when measured from inside the cell, hence the '-'sign.

${ }^{5}$ Since $\beta$ is not the free polymerization rate, for negative curvature (locally concave shape) there could be a slight acceleration of actin polymerization until the free polymerization rate is reached. We neglect this effect here, since it is small and stationary cell shapes are almost exclusively convex, by considering only $c>0$ in the exponential. In the wall energy correction term, however, both curvature signs have to be considered.
} 
relative excess circumference. Note that the latter is not directly restricted via the phase field - only the volume is conserved - but only indirectly via the feedback provided on the polymerization rate. The aspect ratio has been characterized as previously [17], by determining the ratio $h$ of the eigenvalues of the variance matrix $I_{i j}=\int\left(x_{i}-x_{i}^{c}\right)\left(x_{j}-x_{j}^{c}\right) \rho d x d y$, where $\mathbf{r}^{c}=\int \mathbf{r} \rho d x d y$ is the center of mass of the cell. Since a round cell has $h=1$, we treat $h-1$ as a measure for the deviation from a circle.

Figure 1 displays a) the aspect ratio's deviation from the round shape, $h-1, \mathrm{~b})$ the center of mass velocity $V_{\text {com }}$, c) the relative excess circumference $\delta A$ and $\mathrm{d}$ ) the membrane tension $\zeta$, as a function of the membrane's compressibility modulus $T$. Shown are results for two different cells, one of keratocyte shape (black curves) and one of a more fan-like, fibroblast shape (red curves). In case of the first cell type, the aspect ratio expectedly decreases by increasing $T$ : at the sides the curvature is highest, and hence the restoring force leads to a more rounded shape. In contrast, for the second cell type, the aspect ratio increases. This is due to the fan-like, triangular shape of the cell, where membrane tension not only reduces the extension normal to the direction of motion, but also in direction of motion. The velocity is not substantially affected (in the $10-20 \%$ range), in accordance with experiments [25, 24].

The relative excess surface decreases in both cases, cf. Fig. 1c), which shows that in the limit of high $T$, the surface (circumference in 2D) should become rather well conserved, even without explicit inclusion into the phase field equation. Although the relative excess surface decreases, the overall tension $\zeta=T \delta A$ increases, cf. Fig. 1d), as it should. Experimentally, also the membrane tension is accessible by pulling membrane tethers from moving cells, as has been recently studied in [24].

Figure 2 shows the respective shape changes, comparing low and high membrane extensibility moduli (end hence tension values) for the two kinds of cells.

Arrest of cell motion by increased tension. Next we have studied whether the onset/cessation of motion is affected by membrane tension. Mildly driven cells (i.e. cells with not too large values of the propulsion parameter $\alpha$, as well as $\sigma$ and $\gamma$ ) can indeed be stopped by increasing the compresibility modulus $T$ and hence the tension. This effect has been seen in several experiments, e.g. in [22] for leukocytes, and is captured by our model as shown in Fig. 3. Interestingly, while without membrane tension both velocity and aspect ratio exhibit a jump at the arrest of motion, cf. [17], with 
tension present the aspect ratio decreases continuously and the cell becomes even slightly stretched in the direction of motion, before finally being stopped for even higher tension. The reason is that the propulsion is predominantly due to the front-rear asymmetry. Increasing $T$ strongly affects the sides (i.e. the aspect ratio decreases), but the feedback on polymerization is small at the front (and typically also at the rear) since curvature is small there. Consequently, the jump in the velocity is decoupled from the jump in the aspect ratio, due to tension ${ }^{6}$.

Experiments indicated a direct relation between membrane tension and the actin pushing force: reducing the latter results in a decrease in membrane tension, as found by treating keratocytes with blebbistatin and jasplakinolidine, leading to a rapid cessation of the actin assembly [24]. Ref. [22] found the same, namely that membrane tension increases upon stronger leadingedge protrusion. This relation can be directly inferred from our model: namely, it is the propulsion by actin $\propto \alpha$ (and caused by actin polymerization $\propto \beta$ ) that induces an increase in the excess area $\delta A$ (even in the non-motile case) and hence in tension $\zeta=T \delta A / A_{0}$, cf. Eq. (8).

Another interesting experiment performed in Ref. [24] was the fusion of a moving keratocyte with a giant unilammelar vesicle (GUV) to increase the available membrane area. The cross-sections of the cells were found to become larger (i.e. volume entered from the third dimension) with higher aspect ratios, while the velocity and tension remained the same. This behavior too is in accordance with our model: after fusing a cell with a GUV, both the contact area $V_{0}$ and the circumference $A_{0}$ will increase due to spreading. We already studied the behavior of velocity and aspect ratio as a function of the cell's contact area in [17], and found that (keeping all other parameters fixed) for not too large cells the aspect ratio increases with area while the velocity remained practically unchanged. Membrane tension will not change this scenario due to the concomitant increase in $A_{0}$ (the restoring force may decrease a little, since it is proportional to curvature, but the leading edge is rather straight anyways).

In Ref. [24] it was also observed that an increase in cellular adhesion

\footnotetext{
${ }^{6}$ Note that the study in Ref. [17] indicated that there is not a simple relation between the aspect ratio and the center of mass velocity, e.g. when varying the cell's contact area (volume in 3D); here the same is true even for fixed contact area, but for different values of tension. Hence again, the aspect ratio is not necessarily related in a simple way to the cell's velocity.
} 
to the underlying substrate increased the membrane tension. Again, this is consistent with our model since the propulsion force is proportional to the number of adhesive ligands $A, \alpha \simeq \alpha_{0} A$, see Ref. [31] for a generalization of the model including explicit adhesion dynamics. Consequently, upon increased adhesion the cell can spread more efficiently, thereby increasing the relative excess area and consequently the tension $\delta A T$.

Finally, Ref. [24] found that a decrease of myosin contraction leads to higher tension. This is the only trend not (yet) captured in our model: if the parameters associated with the activity of motors $(\sigma$ or $\gamma)$ are decreased, we do obtain more round shapes, but this is not due to an increase in tension. The reason for this discrepancy might well be that the implementation of the action of motors is still too oversimplified: we neither implemented explicit motors, nor tensorial active stresses, nor the contractile bundle at the rear present in keratocytes.

\section{Contact area vs. contour conservation}

There arises the question, which quantity should be conserved in an effective 2D model of a cell. For a 3D cell this is rather clear: the volume is conserved since the cytoplasm is incompressible, and the membrane area too since the membrane's compressibility modulus is very high, implying almost perfect inextensibility (note, however that there are cells with membrane folds to buffer surface area, see also below). In contrast, for a height-averaged 2D model as ours, both the contact area could vary - e.g. the cell retracts to the third dimension by reducing its spreading - and the circumference.

One can describe this effect on a phenomenological level by considering the phase field parameter $\delta$ to depend on both the contact area and the length of the circumference:

$$
\delta=\frac{1}{2}+\mu_{V}\left[V(t)-V_{0}\right]+\mu_{A} T\left[A(t)-A_{0}\right] .
$$

Here, we tuned $\mu_{V}$ and $\mu_{A}$ in such a way that for a moderate value of the compressibility modulus, $T=50$, both contributions are of same order (for a cell of specific size; we typically used cell's of radius $\left.r_{0}=15\right)$. Hence $T=0$ corresponds to pure contact area conservation, while the limit $T \gg 50$ leads to a dominating conservation of the circumference.

We investigated the model described by Eqs. (10) and (11), with the parameter $\delta$ replaced by Eq. (12). Figure 4 displays the aspect ratio, the center 
of mass velocity, the contact area $\delta V$, and the circumference $\delta A$, as a function of the membrane's compressibility modulus $T$. Expectantly, with combined area-circumference conservation, the circumference is better conserved at the cost of the contact area conservation [see panels c) and d)]. Figures 4a) and b) show that the effect of tension on both the aspect ratio and the velocity are much smaller than when only area conservation constraint is imposed. Hence interestingly, although one would naively expect a decrease of speed from the exponential decrease of polymerization with tension, the speed remains almost unchanged due to the global shape dynamics: one can not conclude the overall speed just from the local polymerization rate. In case of only contact area conservation, the velocity decreased by 10-20\%, cf. also Fig. 1. In contrast, if the circumference conservation is important, the overall decrease is below $5 \%$ and probably not even measurable in experiments. Note that keratocytes are known to have only transient, weak adhesion and high membrane tension [24], hence circumference conservation should be important. Indeed both experiments $[25,24]$ did not see an effect of membrane tension on the cell's velocity. We are not aware of such experiments on fibroblasts, but given their higher adhesion and lower tension, the study in the previous section suggests that their velocity might be affected in the 10-20\% range.

Thus, our study indicates that the exchange of contact area and membrane circumference with the third dimension is crucial for understanding the influence of membrane tension on the shape and speed of cells. Obviously, this problem can only be properly addressed in truly three-dimensional models which are underway [34]. Note that the membrane's curvature in this third direction is typically much higher than the in-plane one (due to the thinness of the lamellipodium) and might be the dominating curvature [49]. Another complication arises due to the existence of plasma membrane reservoirs. While these reservoirs seem not to be present in keratocytes [24], they are known to exist for neutrophils (where they are important for phagocytosis [50], i.e. the uptake of micron sized objects), as well as in fibroblasts. The percentage of area buffered/stored can be between 1\% for strongly adhering, up to $10 \%$ for spreading fibroblasts $[23,51]$.

\section{Effect of bending rigidity \& perturbing moving cells}

The bending rigidity contribution to the force opposing actin polymerization is typically negligible compared to the contribution due to tension (cf. Section 3 and Appendix). Nevertheless, bending may affect the shape of 
the cell directly when its contribution to the phase field equation becomes relevant. From the typical $2 \mathrm{D}$ radii of curvature (of order $20 \mu \mathrm{m}$ ) and typical membrane rigidities, one expects only moderate effects for stationary moving cells - mostly at the wings where the curvature is the highest. However, bending may well be relevant for the dynamics of cells and for the response to external perturbations, e.g. via modulations of the substrate properties as studied in $[31,32]$ or by obstacles.

For vesicles, the bending energy can be introduced similar as the phase field energy in Eq. (1), by considering the already introduced function $\lambda=$ $D_{\rho} \Delta \rho-f^{\prime}(\rho)$. Helfrich-Willmore theory [52] then implies the bending energy to be $[37]$

$$
E_{b}=\frac{6 \sqrt{2}}{D_{\rho} \sqrt{D_{\rho}}} b \int \lambda^{2} d x d y
$$

In the sharp interface limit, see Ref. [53], this expression tends to $b \int C_{M}^{2} d x d y$, where $C_{M}$ is the mean curvature and $b$ is the bending modulus. However, this approach can only be used in the 'advected field' case, i.e. if the shape of the phase field $\rho$ across the interface stays (close to) a tanh-profile all the time. This is the case for vesicles in Stokes flow, and also in the active gel hydrodynamics approach recently studied [37], since there are no forces normal to the interface. In the model for a moving cell considered here, however, the active terms (proportional to $\alpha$ and $\sigma$ ) are normal forces and deform the interface away from the radial tanh-shape. We checked the overall bending energy using Eq. (13) for stationary round cells. We obtained that for $\alpha \gtrsim 0.5$ - already well below values needed to induce motility - the bending energy not only increased in value, but, in addition, did not display anymore the correct behavior $E_{b} \propto \frac{b}{R}$. Hence in Eq. (13) one would have to correct for the terms perturbing the radial profile of the phase field, which is neither a straightforward task nor very intuitive.

We therefore applied a purely 2D formulation for the bending energy [47] given by Eq. (6), i.e. used

$$
E_{b}=\frac{b}{2} \int c^{2}|\nabla \rho| d x d y
$$


leading to the following contribution in the phase field ${ }^{7}$

$$
\partial_{t} \rho=\ldots+b\left\{\frac{c^{3}}{2}+\mathbf{t} \cdot \nabla(\mathbf{t} \cdot \nabla c)\right\}|\nabla \rho| .
$$

As expected, for steady moving cells the effect of bending is not very noticeable, except for very low tension values and rather strongly elongated cells. Figures 5a) and b) show steady moving cells with $T=10$, and $b=0$ and 1 , respectively. One can see that bending leads to a rounding of the wings and slightly also at the back.

We also investigated collisions of cells with rigid round obstacles, to test whether perturbations externally imposing a curvature lead to stronger effects. For this purpose, the obstacle was implemented by a second stationary phase field $\rho_{o}(x, y)$ (that had been relaxed towards a radial tanh-profile of equal width as the stationary cell) and coupled to the cell via a steric interaction energy (as developed previously for multiple cells [35])

$$
E_{\text {steric }}=\frac{1}{2} k \rho^{2} \rho_{o}^{2} \quad \rightarrow \quad \partial_{t} \rho=\ldots-k \rho_{o}^{2} \rho .
$$

For the interaction strength we chose $k=10$, typically, to prevent overlap. Figures 5b)-d) shows a collision with an obstacle of radius $R_{o}=10$ for a cell with low tension, $T=10$, and high bending rigidity, $b=1$. For comparison, Figures 5e)-h) display a cell with the same bending rigidity but higher tension $T=50$. One can clearly see that the cell with low tension becomes very deformed in spite of its bending rigidity, while the second one much less. The second cell relaxes rapidly to its initial shape, albeit deflected in its direction of motion, cf. also Suppl. Movies $1 \& 2$.

Finally, we also studied cells encountering a narrow channel, a situation increasingly studied experimentally [54], cf. also [55]. Expectantly, for large enough channel widths the cells pass, while narrow channels stop them at the entrance. Suppl. Movies $3 \& 4$ focus on the intermediate behavior: Movie 3 shows a cell with low tension, $T=10$, that is able to go through the channel albeit being slowed down. Movie 4, in contrast, shows a cell with

\footnotetext{
${ }^{7}$ Since the bending contributions scale with the radius of curvature like $1 / R^{3}$ and rapidly grow towards the inside of the cell, for numerical reasons one has to decrease $D_{\rho}$ slightly (increasing the sharpness of the interface) and also the tube width around the interface where the terms are calculated.
} 
high tension, $T=50$, encountering the same channel. It is less deformable and hence does not pass.

We would like to stress that the studies on external cell perturbations discussed here are so far only preliminary. Nevertheless they already highlight the versatility of the approach, as well as demonstrate possible effects of the membrane on the overall deformability of the cell.

\section{Conclusions}

We investigated the consequences of a variety of physical mechanisms associated with the cell membrane, such as the feedback of membrane tension on actin polymerization, contact area vs. circumference conservation, and membrane bending stiffness, on the shape and motility of moving cells. These questions are important in the context of cell motility, since especially the membrane tension acts as a global mechanical feedback that may constitute a conduit for rapid - note that tension relaxes on the time scale of few milliseconds - information transfer across the cell. In addition, physical perturbations of cells, by e.g. collisions or substrate modulations, are increasingly investigated. The overall conclusion is that the membrane plays an important role in preserving the integrity of the cell as well as for its dynamical response.

We have found that the dominant effect of the membrane on the cell's motility is its feedback via tension on the ratcheting of actin. This is in accordance with the finding by Lieber et al. [24] that for keratocytes, membrane tension is dominated by the cytoskeletal forces. Since keratocytes have rather high tension compared to other moving cells, the most appropriate model for this cell type is the one with combined contact area and circumference conservation, developed in section 4. Accordingly, bending only leads to small corrections, mostly during cellular reshaping events.

Interesting future aspects of membrane effects include generalizations to 3D models of cells. There, the high out-of-plane curvature at the tip of the lamellipodium may dominate the behavior [49]. Another important aspect is related to the fact that the tension originates not only from the lipid bilayer membrane, but also from the actin cortex directly underneath, adhering to the membrane. A loss of the membrane-cytoskeleton adhesion then can lead to blebbing [56], constituting another means of motility, which has not yet been modeled in a whole cell, dynamical model. 


\section{Acknowledgments}

B.W. and F.Z. acknowledges funding from the German Science Foundation (DFG) via project ZI 1232/2-1. I.S.A. was supported by the US Department of Energy (DOE), Office of Science, Basic Energy Sciences (BES), Materials Science and Engineering Division. The numerical work was in part performed on the Northern Illinois University GPU cluster GAEA.

\section{Appendix: Parameters and estimate of tension vs. bending}

The typical scales in our model are chosen to be seconds for time, microns for length and piconewtons for force (see [17] for details).

The force given in Eq. (7) was derived for vesicles [47] and is a volume force (entering the Stokes equation), i.e. it has units of $\mathrm{N} / \mathrm{m}^{3}$. To obtain the force acting on an actin filament, we hence have to multiply by the volume of an actin monomer, roughly $a^{3}=(10 \mathrm{~nm})^{3}$. In the feedback on the

polymerization rate, in the expression $\exp \left(\frac{a F}{k_{B} T}\right)$ one also has $a=10 \mathrm{~nm}$ and $k_{B} T \simeq 4 \mathrm{pNnm}$.

The membrane's compressibility modulus is of order $T=0.1 \mathrm{~J} / \mathrm{m}^{2}[21]$, leading to $10^{5} \mathrm{pN} / \mu \mathrm{m}$. For numerical reasons we varied $T=0-500$. The tension $\zeta$ should be of the same order [21]; it has been recently measured in [24] by pulling membrane tethers from moving keratocytes, and was found to be of the order of $250 \mathrm{pN} / \mu \mathrm{m}$. The force due to membrane tension can be hence estimated to be $\zeta c|\nabla \rho| a^{3} \simeq 0.01-1 \mathrm{pN}$, depending on whether one uses the typical phase field interface width $(\simeq 1 \mu \mathrm{m})$ or rather a real membrane thickness $(5 \mathrm{~nm})$. Consequently, the prefactor $f_{0}$ in $\exp \left(-f_{0} \zeta c\right)$ occurring in Eq. (11) should be of order $0.01-1$, we typically used 1.

In contrast, for the bending rigidity contribution in Eq. (7) we obtain $b c^{3}|\nabla \rho| a^{3}=10^{-5}-10^{-7} \mathrm{pN}$, using a typical membrane bending modulus of order of $b=100 k_{B} T=0.4 \mathrm{pN} \mu \mathrm{m}$. In [24] also the bending rigidity has been directly measured for keratocytes and found to be slightly lower, $0.14 \mathrm{pN} \mu \mathrm{m}$. Thus, for the membrane's feedback on the polymerization rate, bending is completely negligible vs. tension. In the phase field equation, however, the bending contribution enters directly i.e. is of order $0.14-0.4$ in our units, we typically used $b=0-1$. 


\section{References}

[1] T. J. Mitchison, L. P. Cramer, Actin-based cell motility and cell locomotion, Cell 84 (1996) 371.

[2] C. W. Wolgemuth, Does cell biology need physicists?, Physics 4 (2011) 4 .

[3] A. Mogilner, Mathematics of cell motility: have we got its number?, J. Math. Biol. 58 (2009) 105134.

[4] K. E. Kasza, A. C. Rowat, J. Liu, T. E. Angelini, C. P. Brangwynne, G. H. Koenderink, D. A. Weitz, The cell as a material, Curr. Opin. Cell Biol. 19 (2007) 101.

[5] M. S. Mizuhara, L. Berlyand, V. Rybalko, L. Zhang, On an evolution equation in a cell motility model, arXiv preprint arXiv:1506.03945.

[6] P. Friedl, D. Gilmour, Collective cell migration in morphogenesis, regeneration and cancer, Nature Rev. Mol. Cell Biol. 10 (2009) 445.

[7] J. Banchereau, R. M. Steinman, Dendritic cells and the control of immunity, Nature 392 (1998) 245.

[8] A. B. Verkhovsky, T. M. Svitkina, G. G. Borisy, Self-polarization and directional motility of cytoplasm, Curr. Biol. 9 (1999) 11-20.

[9] B. Szabó, G. J. Szöllösi, B. Gönci, Z. Jurányi, D. Selmeczi, T. Vicsek, Phase transition in the collective migration of tissue cells: Experiment and model, Phys. Rev. E 74 (2006) 061908.

[10] J. Etienne, J. Fouchard, D. Mitrossilis, N. Bufi, P. Durand-Smet, A. Asnacios, Cells as liquid motors. mechanosensitivity emerges from collective dynamics of actomyosin cortex, Proc. Natl. Acad. Sci. USA 112 (2015) 2740.

[11] G. V. Kolmakov, A. Schaefer, I. Aranson, A. C. Balazs, Designing mechano-responsive microcapsules that undergo self-propelled motion, Soft Matter 8 (2012) 180-190. 
[12] G. Mahmud, C. J. Campbell, K. J. Bishop, Y. A. Komarova, O. Chaga, S. Soh, S. Huda, K. Kandere-Grzybowska, B. A. Grzybowski, Directing cell motions on micropatterned ratchets, Nature Phys. 5 (2009) 606.

[13] M. Abercrombie, The crawling movement of metazoan cells, Proc. R. Soc. London B 207 (1980) 129-147.

[14] K. Kruse, J. F. Joanny, F. Jülicher, J. Prost, Contractility and retrograde flow in lamellipodium motion, Phys. Biol. 3 (2006) 130.

[15] C. W. Wolgemuth, J. Stajic, A. Mogilner, Redundant mechanisms for stable cell locomotion revealed by minimal models, Biophys. J. 101 (2011) 545 .

[16] D. Shao, W. J. Rappel, H. Levine, Computational model for cell morphodynamics., Phys. Rev. Lett. 105 (2010) 108104.

[17] F. Ziebert, S. Swaminathan, I. S. Aranson, Model for self-polarization and motility of keratocyte fragments, J. Royal Soc. Interface 9 (2012) 1084 .

[18] P. Recho, L. Truskinovsky, Asymmetry between pushing and pulling for crawling cells, Phys. Rev. E 87 (2013) 022720.

[19] D. Raucher, M. P. Sheetz, Cell spreading and lamellipodial extension rate is regulated by membrane tension, J. Cell Biol. 148 (2000) 127-36.

[20] N. Gauthier, T. Masters, M. Sheetz, Mechanical feedback between membrane tension and dynamics, Trends Cell Biol. 22 (2012) 527.

[21] A. Diz-Munoz, D. Fletcher, O. Weiner, Use the force: membrane tension as an organizer of cell shape and motility, Trends Cell Biol. 23 (2013) 47 .

[22] A. Houk, A. Jilkine, C. Mejean, R. Boltyanskiy, E. Dufresne, S. Angenent, S. Altschuler, L. Wu, O. Weiner, Membrane tension maintains cell polarity by confining signals to the leading edge during neutrophil migration, Cell 148 (2012) 175.

[23] N. C. Gauthier, M. A. Fardin, P. Roca-Cusachs, M. P. Sheetz, Temporary increase in plasma membrane tension coordinates the activation of 
exocytosis and contraction during cell spreading, Proc. Natl. Acad. Sci. USA 108 (2011) 1446714472.

[24] A. D. Lieber, S. Yehudai-Resheff, E. L. Barnhart, J. A. Theriot, K. Keren, Membrane tension in rapidly moving cells is determined by cytoskeletal forces, Curr. Biol. 23 (2013) 1409-1417.

[25] T. Fuhs, M. Goegler, C. A. Brunner, C. W. Wolgemuth, J. A. Kaes, Causes of retrograde flow in fish keratocytes, Cytoskeleton 71 (2014) 24-35.

[26] E. M. Craig, D. Van Goor, P. Forscher, A. Mogilner, Membrane tension, myosin force, and actin turnover maintain actin treadmill in the nerve growth cone, Biophys. J. 102 (2012) 1503.

[27] Y. Schweitzer, A. D. Lieber, K. Keren, M. M. Kozlov, Theoretical analysis of membrane tension in moving cells, Biophys. J. 106 (2014) 84.

[28] A. D. Lieber, Y. Schweitzer, M. M. Kozlov, K. Keren, Front-to-rear membrane tension gradient in rapidly moving cells, Biophys. J. 108 (2015) 1599.

[29] B. Fogelson, A. Mogilner, Computational estimates of membrane flow and tension gradient in motile cells, PLoS ONE 9 (2014) e84524.

[30] D. Shao, H. Levine, W.-J. Rappel, Coupling actin flow, adhesion, and morphology in a computational cell motility model, Proc. Natl. Acad. Sci. USA 109 (2012) 6851.

[31] F. Ziebert, I. S. Aranson, Effects of adhesion dynamics and substrate compliance on the shape and motility of crawling cells, PLoS ONE 8 (5) (2013) e64511.

[32] J. Löber, F. Ziebert, I. S. Aranson, Modeling crawling cell movement on soft engineered substrates, Soft Matter 10 (2014) 1365-1373.

[33] F. Ziebert, I. S. Aranson, Modular approach for modeling cell motility, Eur. Phys. J. Special Topics 223 (2014) 1265-1277.

[34] E. Tjhung, A. Tiribocchi, D. Marenduzzo, M. E. Cates, A minimal physical model captures the shapes of crawling cells, Nature Comm. 6 (2015) 5420. 
[35] J. Löber, F. Ziebert, I. S. Aranson, Collisions of deformable cells lead to collective migration, Sci. Rep. 5 (2015) 9172.

[36] E. Tjhung, D. Marenduzzo, M. E. Cates, Spontaneous symmetry breaking in active droplets provides a generic route to motility, Proc. Natl. Acad. Sci. USA 109 (2012) 12381-12386.

[37] W. Marth, S. Praetorius, A. Voigt, A mechanism for cell motility by active polar gels, J. R. Soc. Interface 12 (2015) 20150161.

[38] N. Yoshinaga, Spontaneous motion and deformation of a self-propelled droplet, Physical Review E 89 (2014) 012913.

[39] F. Ziebert, J. Löber, and I. S. Aranson, in Physical Models of Cell Moility, I. S. Aranson, Ed. (Springer ebook, to be published 2015).

[40] C. S. Peskin, G. M. Odell, G. F. Oster, Cellular motions and thermal fluctuations: The brownian ratchet, Biophys. J. 65 (1993) 316.

[41] A. Mogilner, G. Oster, Cell motility driven by actin polymerization, Biophys. J. 71 (1996) 3030-45.

[42] D. R. Kovar, T. D. Pollard, Insertional assembly of actin filament barbed ends in association with formins produces piconewton forces, Proc. Natl. Acad. Sci. USA 101 (2004) 14725.

[43] M. J. Footer, J. W. Kerssemakers, J. A. Theriot, M. Dogterom, Direct measurement of force generation by actin filament polymerization using an optical trap, Proc. Natl. Acad. Sci. USA 104 (2007) 2181.

[44] C. Brangbour, O. du Roure, E. Helfer, D. Démoulin, A. Mazurier, M. Fermigier, M.-F. Carlier, J. Bibette, J. Baudry, Force-velocity measurements of a few growing actin filaments, PLoS Biology 9 (2011) e1000613.

[45] R. Folch, J. Casademunt, A. Hernandez-Machado, L. Ramirez-Piscina, Phase-field model for hele-shaw flows with arbitrary viscosity contrast. i. theoretical approach, Phys. Rev. E 60 (1999) 1724.

[46] T. Biben, C. Misbah, Tumbling of vesicles under shear flow within an advected-field approach, Phys. Rev. E 67 (2003) 031908. 
[47] I. Cantat, C. Misbah, Vesicle dynamics in chemotaxis, haptotaxis, and under shear flow, in: S.C. Müller et al., Ed., Transport and structure, Lecture Notes in Physics 532 (1999) 93-136.

[48] S. Chandrasekhar, Hydrodynamic and hydromagnetic stability, Courier Corporation, 2013.

[49] C. Gabella, E. Bertseva, C. Bottier, N. Piacentini, A. Bornert, S. Jeney, L. Forro, I. F. Sbalzarini, J.-J. Meister, A. B. Verkhovsky, Contact angle at the leading edge controls cell protrusion rate, Current Biol. 24 (2014) 1126 .

[50] M. Herant, V. Heinrich, M. Dembo, Mechanics of neutrophil phagocytosis: experiments and quantitative models, J. Cell Sci. 119 (2006) 1903.

[51] D. Raucher, M. P. Sheetz, Characteristics of a membrane reservoir buffering membrane tension, Biophys. J. 77 (1999) 1992.

[52] Q. Du, C. Liu, R. Ryham, X. Wang, A phase field formulation of the willmore problem, Nonlinearity 18 (2005) 1249.

[53] G. Bellettini, L. Mugnai, On the approximation of the elastica functional in radial symmetry, Calc. Var. Partial Differ. Equ. 24 (2005) 1.

[54] C. G. Rolli, T. Seufferlein, R. Kemkemer, J. P. Spatz, Impact of tumor cell cytoskeleton organization on invasiveness and migration: A microchannel-based approach, PLoS ONE 5 (2010) e8726.

[55] R. J. Hawkins, M. Piel, G. Faure-Andre, A. M. Lennon-Dumenil, J. F. Joanny, J. Prost, R. Voituriez, Pushing off the walls: A mechanism of cell motility in confinement, Phys. Rev. Lett. 102 (2009) 058103.

[56] J. Dai, M. P. Sheetz, Membrane tether formation from blebbing cells, Biophys. J. 77 (1999) 3363. 

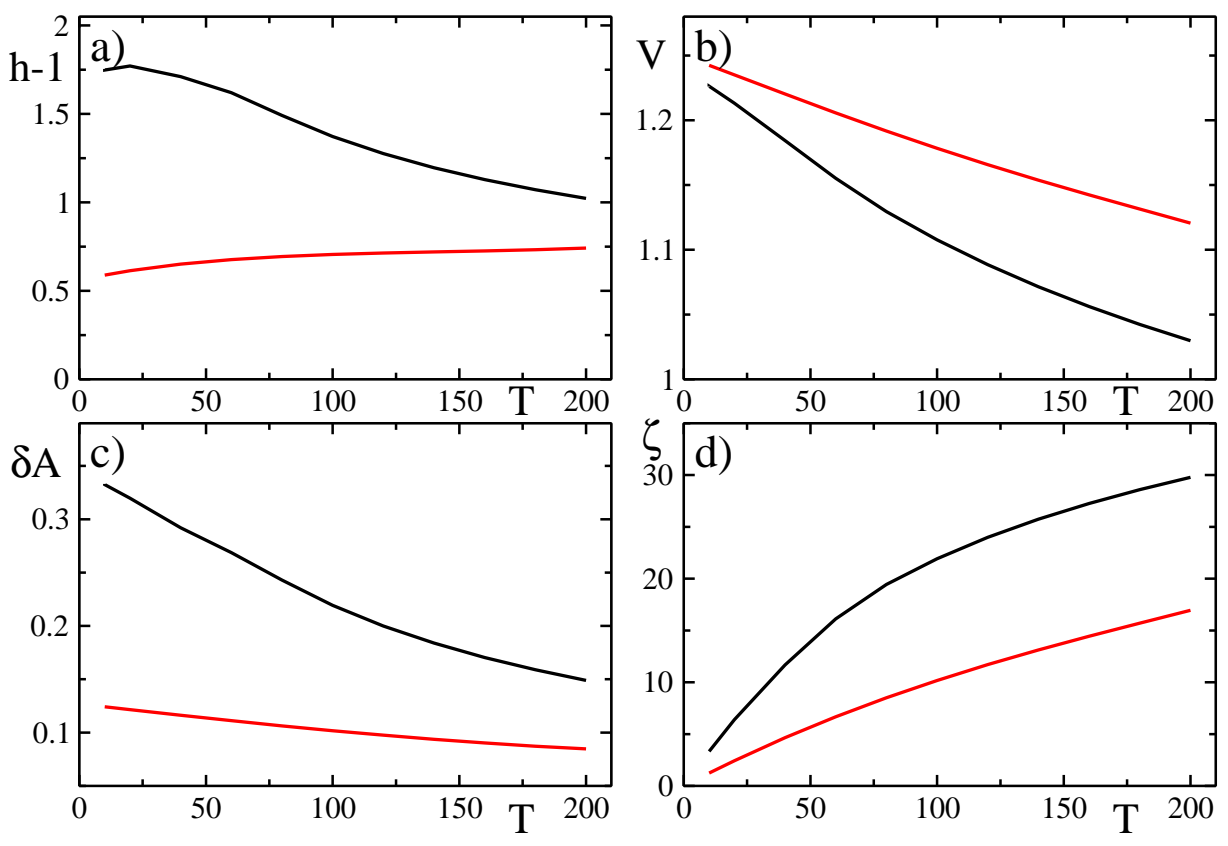

Figure 1: The aspect ratio's deviation from round shape, $h-1$ (a), the center of mass velocity $V_{\text {com }}(\mathrm{b})$, the relative excess circumference $\delta A$ (c), and the membrane tension $\zeta=T \delta A(\mathrm{~d})$, for a keratocyte-like cell (black curves; $\alpha=2, \beta=2, \sigma=1.2, \gamma=0$ ), and for a fibroblast-like cell (red curves; $\alpha=3, \beta=1.5, \sigma=0.9, \gamma=0.3$ ) vs membrane compressibility constant $T$. Remark: the volume is conserved by better then $1 \%$ in both cases. 


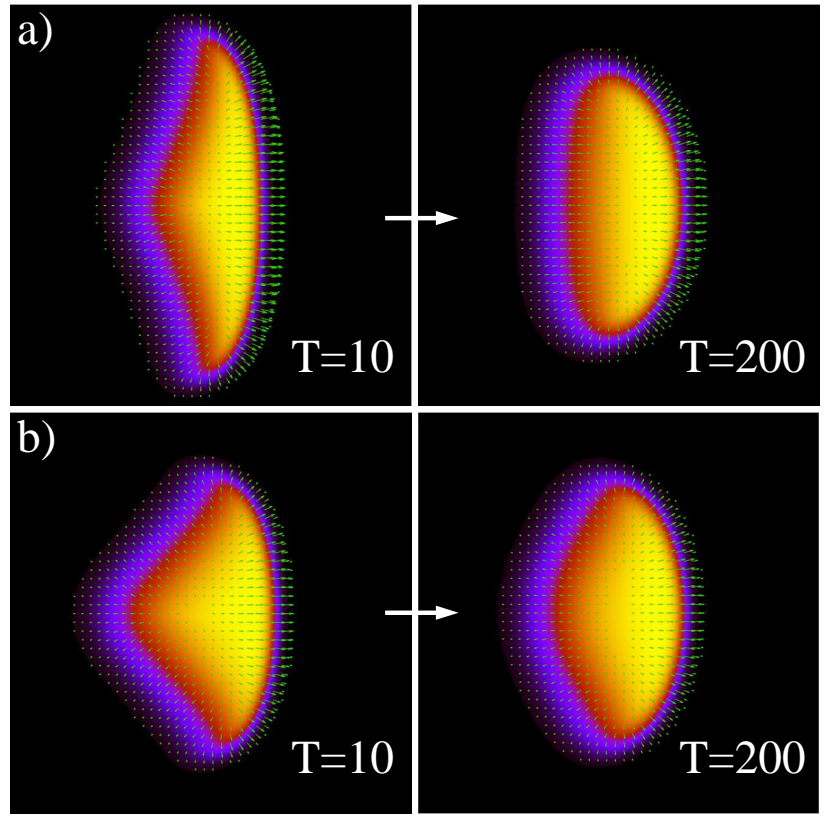

Figure 2: The shapes for a) a keratocyte-like cell $(\alpha=2, \beta=2, \sigma=1.2, \gamma=0)$ and b) a fibroblast-like cell $(\alpha=3, \beta=1.5, \sigma=0.9, \gamma=0.3)$ for small $(T=10)$ and large $(T=200)$ membrane's compressibility modulus, cf. also the curves in Fig. 1.

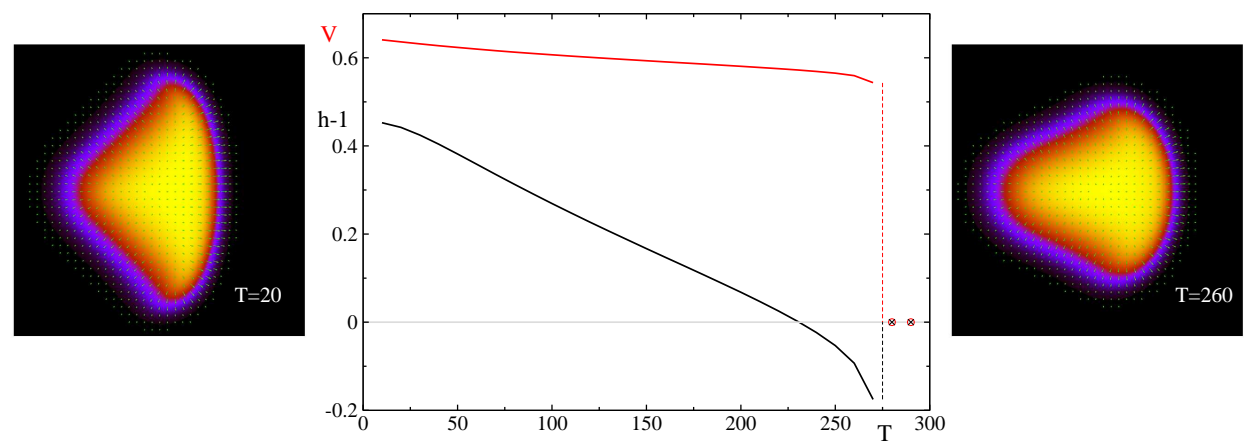

Figure 3: Evolution of the aspect ratio's deviation from round shape, $h-1$, and center of mass velocity $V_{\text {com }}$ for mildly driven cells (parameters: $\alpha=1.3, \beta=1.3, \sigma=1.4, \gamma=0$ ). The tension $T$ was increased step by step and the velocity and aspect ratio measured after the cell had equilibrated to its steady state. At a certain critical value $T_{c}$ the cell stops. The snapshots at the left and right show the cell's shapes for small (negligible) tension and for the highest tension value where the cell still is able to move. 

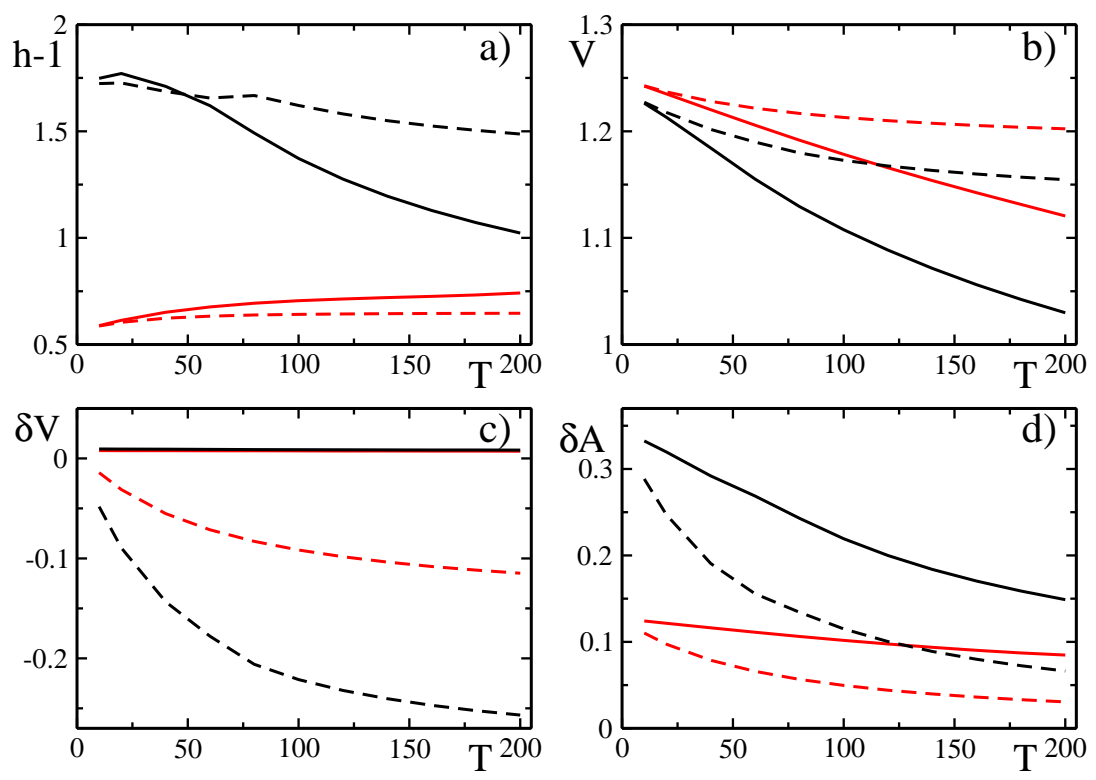

Figure 4: The aspect ratio's deviation from round shape $h-1$ (a), the center of mass velocity $V_{\text {com }}(\mathrm{b})$, the excess contact area $\delta V$ (c), and the relative excess circumference $\delta A$ (d) for two cell types are shown as a function of the membrane's compressibility modulus $T$ : for a keratocyte-like cell $(\alpha=2, \beta=2, \sigma=1.2, \gamma=0)$ with area conservation (black solid) and with combined area-circumference conservation (black dashed), as well as for a fibroblast-like cell ( $\alpha=3, \beta=1.5, \sigma=0.9, \gamma=0.3)$, again with area conservation (red solid) and with combined area-circumference conservation (red dashed). Note that the solid curves are identical to those in Fig. 1. 

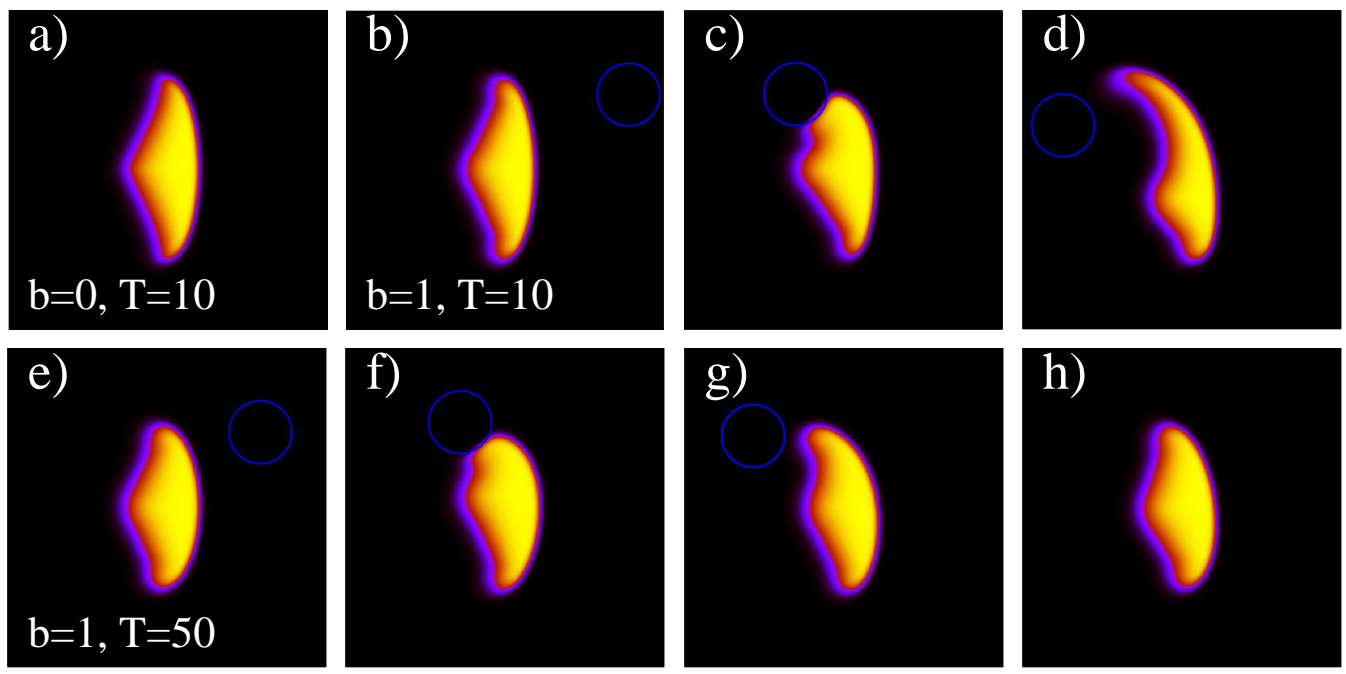

Figure 5: Sequences of snapshots illustrating the collision between a moving cell and an obstacle. Panel a) shows a steady moving cell with low tension $(T=10)$ and no bending. Panels b)-d) display the collision of a cell with the same tension and bending rigidity $b=1$ with a round obstacle. From the steady moving shape b) in comparison to a) one can infer that bending leads to a slight rounding of the wings and the back. For such a 'floppy' cell of low tension, the collision leads to a strong deformation of the wing hitting the obstacle. Parts e)-h) show a collision of a cell with higher tension $T=50$ and same bending rigidity, with an obstacle. The cell is much less deformed, recovers its initial shape more rapidly and is slightly deflected from its initial direction of motion. The collision events have been traced in the moving frame of the cell. Other parameters: $\alpha=\beta=1.3, \sigma=0.6, \gamma=0.4$; $D_{\rho}=0.5$ in place of $D_{\rho}=1$ which was used in the other figures. 